\title{
Article \\ Power Allocation for Reliable and Energy-Efficient Optical LEO-to-Ground Downlinks with Hybrid ARQ Schemes
}

\author{
Theodore T. Kapsis and Athanasios D. Panagopoulos * (D) \\ School of Electrical and Computer Engineering, National Technical University of Athens, \\ GR-15780 Athens, Greece; teokapsis@mail.ntua.gr \\ * Correspondence: thpanag@ece.ntua.gr
}

check for updates

Citation: Kapsis, T.T.; Panagopoulos, A.D. Power Allocation for Reliable and Energy-Efficient Optical

LEO-to-Ground Downlinks with

Hybrid ARQ Schemes. Photonics 2022 9, 92. https://doi.org/10.3390/ photonics 9020092

Received: 15 December 2021

Accepted: 2 February 2022

Published: 4 February 2022

Publisher's Note: MDPI stays neutral with regard to jurisdictional claims in published maps and institutional affiliations.

Copyright: (C) 2022 by the authors. Licensee MDPI, Basel, Switzerland. This article is an open access article distributed under the terms and conditions of the Creative Commons Attribution (CC BY) license (https:// creativecommons.org/licenses/by/ $4.0 /)$.

\begin{abstract}
Satellites in low earth orbit (LEO) are currently being deployed for numerous communication, positioning, space and Earth-imaging missions. To provide higher data rates in direct-to-user links and earth observation downlinks, the free-space optics technology can be employed for LEO-toground downlinks. Moreover, the hybrid automatic repeat request (HARQ) can be adopted since the propagation latency is low for LEO satellites. In this work, a power allocation methodology is proposed for optical LEO-to-ground downlinks under weak turbulence employing HARQ retransmission schemes. Specifically, the average power consumption is minimized given a maximum transmitted power constraint and a target outage probability threshold to ensure energy efficiency and reliability, respectively. The optimization problem is formulated as a constrained nonlinear programming problem and solved for Type I HARQ, chase combining (CC) and incremental redundancy (IR) schemes. The solutions are derived numerically via iterative algorithms, namely interior-point (IP) and sequential quadratic programming (SQP), and validated through an exhaustive (brute-force) search. The numerical simulations provide insight into the performance of the retransmission schemes regarding average power. More specifically, Type I HARQ has the worst output, CC has a moderate one, and IR exhibits the best performance. Finally, the IP algorithm is a slower but more accurate solver, and SQP is faster but slightly less accurate.
\end{abstract}

Keywords: free-space optics; low earth orbit satellite; power allocation; hybrid automatic repeat request; weak turbulence; chase combining; incremental redundancy; outage probability; lognormal fading

\section{Introduction}

As early as the 1960s, the development of optical and laser pumping brought free-space optical (FSO) communication to life [1,2]. Since then, FSO has been employed throughout the industry either for space telecommunication applications such as the modern SpaceX Starlink project for satellite interconnection, optical satellite feeder links and even terrestrial commercial and military applications, e.g., inter-building links [1]. The rollout of the fifth generation (5G) and beyond has especially shifted the research paradigm to optical frequency technologies in order to meet the strict broadband, reliability and latency requirements, leading to the massive installation of fiber optics and optical telescopes [2]. Albeit a relatively new field of communication, FSO systems are considered mature enough to be employed as they hold many noteworthy advantages. More specifically, it is easy to establish point-to-point optical links due to the small-size equipment, lack of any digging, huge bandwidth availability and data rates, unlicensed operation, their strong immunity to unwanted interferences, low-power to ensure eye-safety and improved security due to employment of narrow beams [1-3]. Therefore, assuming good optical signal propagation conditions (clear sky), FSO links guarantee fast, convenient, economic, secure and reliable deployment as well as the efficient usage of the spectrum [1-3].

On the other hand, in the case of atmospheric impairments such as opaque fog and clouds or line-of-sight interruption in general, the irradiance losses can reach hundreds of 
$\mathrm{dBs}$, leading to an optical link outage [1-3]. Moreover, the atmospheric refractive index is not spatially or temporally homogeneous but is varied with wind speed, temperature and wavelengths, which subsequently cause atmospheric turbulence [2-4]. The turbulence in turn influences the slant path propagation, causing scintillation of the received irradiance and beam spreading [2-4]. There are also many more sources of deterioration such as pointing jitter and background noise, but these are beyond the scope of this work [4]. Typical mitigation techniques regarding the physical layer include aperture averaging, adaptive optics, hybrid radio frequency (RF)/FSO and spatial diversity, but there are also upper-layer solutions such as automatic repeat request (ARQ) retransmission schemes [3,4].

In particular, hybrid ARQ (HARQ) combines error detection with error correction by adding redundancy bits to the transmitted frames, and if the message decoding fails, a series of retransmission rounds are performed [5-8]. Depending on whether the erroneous message is discarded or stored, the HARQ is categorized as Type I HARQ or soft combining HARQ, respectively [5-8]. The latter is performed in practice based on the following two methods: chase combining (CC), in which the transmitter sends identical copies of the corrupted frame on each round and the receiver employs maximal ratio combining, whereas in incremental redundancy (IR) the transmitter sends more parity bits on each round, increasing the successful decoding probability [5-8]. The HARQ is superior when reliability and link adaptation are required due to the joint error detection and correction, while costwise it is potentially cheaper. HARQ for optical links has shown good performance and can operate in parallel with the aforementioned physical layer techniques [3].

A low Earth orbit (LEO) satellite operates at an altitude less than $2000 \mathrm{~km}$, has a full period of about two hours and exhibits a latency of a few milliseconds (17 times lower than GEO) [2]. If equipped with an optical transmitter it can provide high-definition data at reduced latency. Several optical LEO-to-ground experiments have been conducted to study the feasibility and obtain measurements for channel characterization and modeling [2,9,10]. In [6] a new HARQ protocol for FSO multi-user systems is proposed, and in [8,11,12] a performance analysis of FSO HARQ systems and estimations of the packet error probability are derived. In [13-15] power allocation strategies for RF HARQ links under Rayleigh fading are reported. The LEO satellite-to-ground links are also used for quantum key distribution (QKD). LEO-to-ground QKD links have also very recently been demonstrated to reach distances up to $1200 \mathrm{~km}$ and key rates up to kbps [16]. The key rate is the exchange rate of polarized photons (encryption keys) over an optical fiber or FSO link. Moreover, a study has been evaluated for QKD performance on a hypothetical constellation with ten satellites in sun-synchronous LEOs that are assumed to communicate over a period of one year with an optical infrastructure (three optical ground stations) located in Greece [17]. The atmospheric effects of turbulence and the background solar radiance have been considered [17].

For optical satellite downlinks with HARQ schemes under weak turbulence, there has not been a power allocation investigation in the literature. In this contribution, a power allocation methodology is proposed for optical LEO-to-ground downlinks under weak scintillation conditions employing HARQ retransmission schemes. The contributions of this work are summarized as follows:

- Three power allocation methodologies based on the Type I HARQ, CC and IR schemes are proposed, and their performances are compared and ranked from best to worst in terms of the average power consumption.

- The energy efficiency and the reliability of the optical links are optimized by formulating the optimization problem as a constrained nonlinear programming problem with an objective function, the average power usage, constraints, the maximum transmitted power and a target outage probability accordingly.

- Only the channel statistics (long-term channel state information) are required to obtain the optimal power allocation strategy and not the instantaneous channel states. 
- $\quad$ The proposed solutions are derived numerically via iterative algorithms, namely interior-point and sequential quadratic programming, and validated through an exhaustive or brute-force search [18,19].

- Simulations are executed for various channel conditions and system settings by simulating a LEO passing over various turbulence intensities and ground weather conditions to investigate the sensitivity of the three HARQ schemes to weak scintillation, path loss and target outage probability. Novel numerical results are reported and commented on.

The remainder of the paper is structured as follows: In Section 2 the LEO-to-ground system model is given along with the necessary FSO theory and assumptions regarding the weak fluctuation model. In Section 3 the optimal power allocation problem under maximum power and outage probability constraints is developed by taking into account the three HARQ schemes, and the proposed solutions are reported. In Section 4 simulations of various scintillation, weather conditions and constraints are obtained using the proposed methodology, and numerical results are derived, compared and commented on. Finally, Section 5 concludes this article and future work is proposed.

\section{System Model}

A single, cloud-free optical LEO-to-ground communication downlink is considered subject to path losses and weak atmospheric turbulence. The optical channel is generally dynamic due to the elevation angle-varying link distance and the LEO satellite's slew rate, thus leading to temporal signal fluctuations known as scintillation [2,9]. For the transmission, the intensity modulation with on-off-keying (OOK) is assumed, and direct detection is used for the reception. It is also hypothesized that a negative acknowledgment (NACK) or no acknowledgment at all to a particular frame transmission by the receiving terminal will initiate a series of retransmissions via an HARQ protocol [5-8]. The maximum number of HARQ rounds is predefined and equal to $M$. During these $M$ rounds, the receiver either successfully decodes the message and responds with a positive acknowledgment (ACK) or fails to decode it, and the re-transmission stops. In order to achieve independent fading states, the minimum retransmission time between rounds must be equal to the coherence time $\tau_{0}(\mathrm{sec})$ of the optical channel. According to the weak turbulence model the coherence time is given by $[9,10]$ :

$$
\tau_{0}=\left(118 \lambda^{-2} \sec \left(90^{\circ}-e\right) \int_{H_{\mathrm{OGS}}}^{H_{\mathrm{LEO}}} C_{n}^{2}(\mathrm{z}) V^{5 / 3}(\mathrm{z}) \mathrm{dz}\right)^{-3 / 5},
$$

where $\lambda(m)$ is the communication wavelength, $\sec (x)$ is the secant function, $e(\operatorname{deg})$ is the elevation angle, $H_{\mathrm{OGS}}(m), H_{\mathrm{LEO}}(m)$ are the altitudes of the optical ground station (OGS) and LEO satellite, $C_{n}^{2}(z)$ is the refractive index structure parameter usually given by the Hufnagel-Valley model, $z(m)$ is the altitude, and $V(z)(\mathrm{m} / \mathrm{s})$ is the wind speed (vertical path) usually described by the Bufton model. Therefore, for transmission periods greater than $\tau_{0}$ the fading states can be considered uncorrelated. In Figure 1, the system model is depicted.

Weak turbulence is often represented by the lognormal (LN) distribution, which fits well and exhibits good agreement with first-order statistics from experimental data [2,20]. The $L N$ model does not fully apply only for elevation angles $<20^{\circ}$, and the reason is the saturation of scintillation [2]. The normalized received irradiance $I\left(W / m^{2}\right)$ is an $L N$ random variable according to the following probability density function (PDF) $[4,20]$ :

$$
p(I)=\frac{1}{I \sqrt{2 \pi} \sigma_{I}} \exp \left\{-\frac{\left[\ln (I /\langle I\rangle)+0.5 \sigma_{I}^{2}\right]^{2}}{2 \sigma_{I}^{2}}\right\},
$$


where $\langle I\rangle$ is the average irradiance, and $\sigma_{I}^{2}$ is the so-called scintillation index (SI). A theoretical expression for SI in the case of weak turbulence $(\mathrm{SI}<0.5)$ and point receivers is derived from Rytov, which is expressed by the formula $[9,20]$ :

$$
\sigma_{I}^{2}=2.25 k^{\frac{7}{6}} \sec ^{\frac{11}{6}}\left(90^{\circ}-e\right) \int_{H_{\mathrm{OGS}}}^{H_{\mathrm{LEO}}} C_{n}^{2}(\mathrm{z})\left(\mathrm{z}-H_{\mathrm{OGS}}\right)^{\frac{5}{6}} \mathrm{dz},
$$

where $k(\mathrm{rad} / \mathrm{m})$ is the wavenumber, $e(\mathrm{deg})$ is the elevation angle, and the rest of the parameters are defined as in (1). The Kolmogorov spectrum is assumed, and it is also assumed that the optical wavefront is approximated by a plane wave far from the source. For moderate (SI 0.5) or strong turbulence (SI 1), other distributions such as gammagamma are more suitable [2]. If experimental data are to be employed then SI is simply the normalized variance of $I: \sigma_{I}^{2}=\left\langle I^{2}\right\rangle /\langle I\rangle^{2}-1[9,20]$.

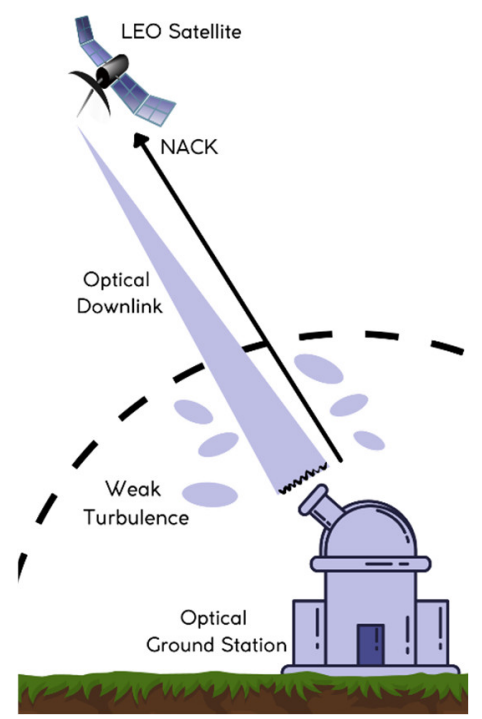

Figure 1. Optical LEO-to-ground downlink. The atmospheric turbulence attenuates the signal, distorts the wavefront and induces scintillation.

The optical channel for the downlink follows the $L N$ distribution considering weak scintillation conditions. By incorporating the quantum efficiencies of the transmitter $\eta_{\text {LEO }}$ and receiver $\eta_{\mathrm{OGS}}$, the atmospheric transmittance $T_{A t m}$, the gains of transmitter $g_{\mathrm{LEO}}$ and receiver $g_{\mathrm{OGS}}$, the large-scale path loss $P L$, the small-scale loss due to scintillation $e^{2 X_{s}}$ where $X_{s}$ is the log-amplitude of the optical wave and hence Gaussian (normally) distributed, the optical channel is expressed by $[4,21]$ :

$$
h_{o p t}=\eta_{\mathrm{LEO}} \eta_{\mathrm{OGS}} T_{A t m} g_{\mathrm{LEO}} g_{\mathrm{OGS}} P L e^{2 X_{s}}
$$

Additionally, therefore, the signal-to-noise ratio (SNR) is given by $[4,21]$ :

$$
S N R=\frac{P_{T} \eta_{\mathrm{LEO}} \eta_{\mathrm{OGS}} T_{A t m} g_{\mathrm{LEO}} g_{\mathrm{OGS}} P L e^{2 X_{s}}}{\sigma_{n_{o p t}}^{2}}=\frac{P_{T} h_{o p t}}{\sigma_{n_{o p t}}^{2}}=P_{T} h
$$

where $P_{T}$ is the transmitted power, $\sigma_{n_{o p t}}^{2}$ is the signal-independent optical noise variance, and $h\left[\right.$ Watts $\left.^{-1}\right]$ represents the ratio $h_{\text {opt }} / \sigma_{n_{o p t}}^{2}$.

In particular, the optical noise is a zero mean, a constant variance random variable that describes the environmental optical interference. It incorporates the background radiation from the various celestial bodies, the amplified spontaneous emission (ASE) from optical preamplifiers and the electronic detection noise [4,21]. By using narrow passband optical 
filters and small field-of-view telescopes the receiver can eliminate the ambient background radiation and ASE, while the thermal and shot noises can be considered as additive white Gaussian noise [2]. Now, for a fixed $\sigma_{n_{o p t}}^{2}$ it is easy to see that $h$ is also a $L N$ variable, and its PDF is derived from (2) with parameters $\langle h\rangle, \sigma_{h}^{2}=\sigma_{I}^{2}$.

\section{Power Allocation Problem and Methodology}

\subsection{Power Allocation Problem Formulation}

In this subsection, the power allocation problem for an HARQ optical LEO-to-OGS downlink is formulated under a maximum transmitted power constraint and a guaranteed outage probability constraint. Specifically, assuming $M$ rounds of retransmissions via Type I, CC, IR HARQ schemes the optimization problem considers the minimization of the average total transmitted power:

$$
\begin{gathered}
\underset{P_{1}, P_{2}, \ldots, P_{M}}{\operatorname{argmin}} P_{\text {Avg }} \\
\text { s.t. } 0 \leq P_{m} \leq \bar{P}, \text { for } 1 \leq m \leq M \\
0 \leq P_{\text {out }, M}\left(C_{\text {out }}\right) \leq \varepsilon_{\text {target }},
\end{gathered}
$$

where $P_{i}(i=1, \ldots, M)$ is the transmitted power at each round, $\bar{P}$ is the peak transmitted power, $C_{\text {out }}$ is the defined outage capacity, $P_{\text {out }, M}$ is the decoding failure probability after all the $M$ re-transmissions, and $\varepsilon_{\text {target }}$ is the target outage probability. The $P_{\text {out }, m}$ is analyzed below for the three HARQ schemes individually.

The $P_{\text {Avg }}$ for all three investigated HARQ schemes is reported as follows [13-15]:

$$
P_{\text {Avg }}=P_{1}+P_{2} \cdot P_{\text {out }, 1}+\ldots+P_{M} \cdot P_{\text {out }, M-1}=\sum_{m=1}^{M} P_{m} \cdot P_{\text {out }, m-1}
$$

Note that $P_{\text {out }, 0}=1$ because no transmission is achieved at round $m=0$. Successful decoding occurs when the channel capacity on the $m$ th round $C_{m}>C_{\text {out }}$; otherwise, a re-transmission is requested $[13,14]$. After $M$ failures, the buffer empties, and the source proceeds to the next packet. In our analysis, the bit error rate (BER) is not included in (7) because it is complex and requires specific knowledge of coding and modulation. The BER performance for a variety of binary modulations can be found in [12].

\subsubsection{Type I HARQ}

In a Type I HARQ scheme, the received packets are not buffered but discarded on each round. Thus, after the $m$ th round the channel capacity and outage probability are $[5,15]$ :

$$
\begin{gathered}
C_{m}=W \log _{2}\left(1+h_{m} P_{m}\right)(\text { bps }) \\
P_{r}\left(C_{m} \leq C_{\text {out }}\right)=P_{r}\left(h_{m} \leq \frac{2^{\frac{C_{\text {out }}}{W}}-1}{P_{m}}\right)=\frac{1}{2} \operatorname{erfc}\left(\begin{array}{c}
\ln \left(\frac{2^{\frac{C_{\text {out }}}{W}}-1}{\left\langle h_{m}\right\rangle P_{m}}\right)+0.5 \sigma_{h}^{2} \\
\sigma_{h} \sqrt{2}
\end{array}\right) \\
P_{\text {out }, m}=P_{r}\left(C_{1} \leq C_{\text {out }}, \ldots, C_{m} \leq C_{\text {out }}\right)=\prod_{i=1}^{m} P_{r}\left(C_{i} \leq C_{\text {out }}\right),
\end{gathered}
$$

where $W$ is the allocated bandwidth, and $\operatorname{erfc}(\mathrm{x})$ is the complementary error function.

For example, in the case of $M=3$ rounds then the optimization problem is the following:

$$
\underset{P_{1}, P_{2}, P_{3}}{\operatorname{argmin}} P_{1}+P_{2} \cdot \frac{1}{2} \operatorname{erfc}\left(-\frac{\ln \left(\frac{2 \frac{c_{\text {out }}^{W}}{h_{1}}-1}{\left\langle h_{1}\right\rangle P_{1}}\right)+0.5 \sigma_{h_{1}}^{2}}{\sigma_{h_{1}} \sqrt{2}}\right)+P_{3} \cdot \frac{1}{4} \operatorname{erfc}\left(-\frac{\ln \left(\frac{2 \frac{c_{\text {out }}}{W}-1}{\left\langle h_{1}\right\rangle P_{1}}\right)+0.5 \sigma_{h_{1}}^{2}}{\sigma_{h_{1}} \sqrt{2}}\right) \cdot \operatorname{erfc}\left(-\frac{\ln \left(\frac{2 \frac{C_{\text {out }}}{W}-1}{\left\langle h_{2}\right\rangle P_{2}}\right)+0.5 \sigma_{h_{2}}^{2}}{\sigma_{h_{2}} \sqrt{2}}\right)
$$




$$
P_{\text {out }, 3}=\frac{1}{8} \operatorname{erfc}\left(-\frac{\ln \left(\frac{2 \frac{C_{\text {out }}}{W}-1}{\left\langle h_{1}\right\rangle P_{1}}\right)+0.5 \sigma_{h_{1}}^{2}}{\sigma_{h_{1}} \sqrt{2}}\right) \cdot \operatorname{erfc}\left(-\frac{\ln \left(\frac{2 \frac{C_{\text {out }}}{W}-1}{\left\langle h_{2}\right\rangle P_{2}}\right)+0.5 \sigma_{h_{2}}^{2}}{\sigma_{h_{2}} \sqrt{2}}\right) \cdot \operatorname{erfc}\left(-\frac{\ln \left(\frac{2 \frac{C_{\text {out }}}{W}-1}{\left\langle h_{3}\right\rangle P_{3}}\right)+0.5 \sigma_{h_{3}}^{2}}{\sigma_{h_{3}} \sqrt{2}}\right)
$$

\subsubsection{Chase Combining HARQ}

In a CC scheme, the received packets are buffered and MRC-combined on each round. Thus, after the $m$ th round the channel capacity and outage probability are $[5,14]$ :

$$
\begin{gathered}
C_{m}=W \log _{2}\left(1+\sum_{i=1}^{m} h_{i} P_{i}\right)(\text { bps }) \\
P_{r}\left(C_{m} \leq C_{\text {out }}\right)=P_{r}\left(W \log _{2}\left(1+\sum_{i=1}^{m} h_{i} P_{i}\right) \leq C_{\text {out }}\right)=P_{r}\left(h_{1} P_{1}+\ldots+h_{m} P_{m} \leq 2^{\frac{C_{\text {out }}}{W}}-1\right) \\
P_{\text {out }, m}=P_{r}\left(C_{1} \leq C_{\text {out }}, \ldots, C_{m} \leq C_{\text {out }}\right)=P_{r}\left(C_{m} \leq C_{\text {out }}\right)
\end{gathered}
$$

For example, in the case of $M=3$ rounds then the optimization problem is the following:

$$
\begin{gathered}
\underset{P_{1}, P_{2}, P_{3}}{\operatorname{argmin}} P_{1}+P_{2} \cdot \frac{1}{2} \operatorname{erfc}\left(-\frac{\ln \left(\frac{\left.\frac{2 \frac{C_{\text {out }}}{W}-1}{\left\langle h_{1}\right\rangle P_{1}}\right)+0.5 \sigma_{h_{1}}^{2}}{\sigma_{h_{1}} \sqrt{2}}\right)+P_{3} \cdot P_{r}\left(h_{1} P_{1}+h_{2} P_{2} \leq 2^{\frac{C_{\text {out }}}{W}}-1\right)}{P_{\text {out }, 3}=\int_{h_{1}=0}^{\frac{2 \frac{C_{\text {out }}}{W}}{P_{1}}} \frac{\int_{h_{2}=0}^{\frac{C_{\text {out }}}{W}-1-h_{1} P_{1}}}{\int_{P_{2}}^{\frac{C_{\text {out }}}{W}-1-h_{2} P_{2}-h_{1} P_{1}}}} \int_{h_{3}=0}^{P_{3}} \frac{1}{h_{1} h_{2} h_{3} \sigma_{h_{1}} \sigma_{h_{2}} \sigma_{h_{3}} \sqrt[3]{2 \pi}} \mathrm{e}^{(-\mathrm{K}(h)) \mathrm{dh}_{1} \mathrm{dh}_{2} \mathrm{dh}_{3}}\right.
\end{gathered}
$$

where

$$
\mathrm{K}(h)=\frac{\left(\ln h_{1} /\left\langle h_{1}\right\rangle+0.5 \sigma_{h_{1}}^{2}\right)^{2}}{2 \sigma_{h_{1}}^{2}}+\frac{\left(\ln h_{2} /\left\langle h_{2}\right\rangle+0.5 \sigma_{h_{2}}^{2}\right)^{2}}{2 \sigma_{h_{2}}^{2}}+\frac{\left(\ln h_{3} /\left\langle h_{3}\right\rangle+0.5 \sigma_{h_{3}}^{2}\right)^{2}}{2 \sigma_{h_{3}}^{2}}
$$

\subsubsection{Incremental Redundancy HARQ}

In an IR scheme, the received packets are buffered, and the information is added on each round because the packets contain new parity bits. Thus, after the $m$ th round the channel capacity and outage probability are $[5,13]$ :

$$
\begin{gathered}
C_{m}=\sum_{i=1}^{m} W \log _{2}\left(1+h_{i} P_{i}\right)(\text { bps }) \\
P_{r}\left(C_{m} \leq C_{\text {out }}\right) \Rightarrow P_{r}\left(\sum_{i=1}^{m} W \log _{2}\left(1+h_{i} P_{i}\right) \leq C_{\text {out }}\right) \Rightarrow P_{r}\left(\left(1+h_{1} P_{1}\right) \cdots\left(1+h_{m} P_{m}\right) \leq 2^{\frac{C_{\text {out }}}{W}}\right) \\
P_{\text {out }, m}=P_{r}\left(C_{1} \leq C_{\text {out }}, \ldots, C_{m} \leq C_{\text {out }}\right)=P_{r}\left(C_{m} \leq C_{\text {out }}\right)
\end{gathered}
$$

For example, in the case of $M=3$ rounds then the optimization problem is the following:

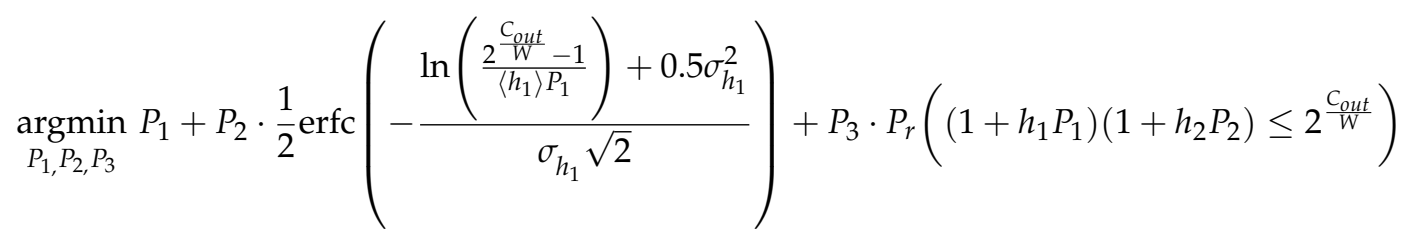




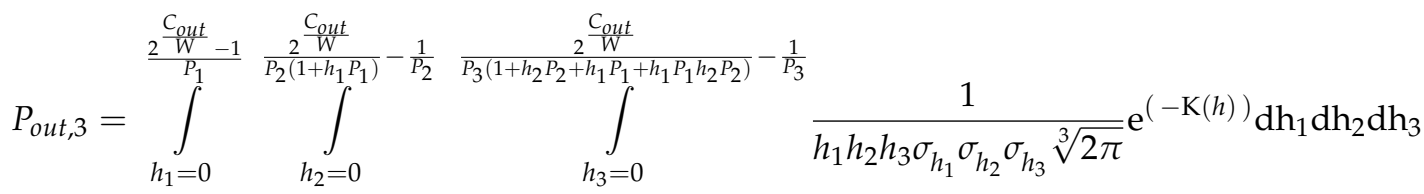

where $\mathrm{K}(h)$ is defined in (19).

The (16) and (22) are based on the fact that in chase combining and incremental redundancy, the channel capacity $C_{m}$ at the end of $m$ th round is non-decreasing for all fading-sequence realizations because the packets are soft-combined $[13,14]$. That is: $C_{1} \leq C_{2} \leq \ldots \leq C_{m}$. Therefore, if $C_{m} \leq C_{\text {out }}$ it means that all the previous rounds' capacities are also less than $C_{\text {out }}$.

The outage probabilities in (18) and (24) are intractable and cannot be solved in closedform, but even numerical computations are challenging for a large $M$. In this work, for the sake of simplicity, we will investigate the scenario with $M=3$ rounds of re-transmission.

\subsection{Power Allocation Methodology}

From the aforementioned analysis in Section 3.1, it was observed that both the objective function and the constraints are non-linear and non-convex. The finding of a global minimum is then NP-hard because it may exist in many feasible regions and many local minima, so a global solution is very difficult to obtain. However, according to the Weierstrass theorem, if the objective function is continuous and the feasible region is closed and bounded, then there exists a global optimum [18,19]. All three HARQ power allocation subproblems satisfy these requirements, and the constraints can be plotted to determine if the feasible region is closed and bounded. The global minimum can always be found then with an exhaustive/brute-force search [13].

In spite of the lack of convexity, the Karush-Kuhn-Tucker conditions are necessary (but not sufficient) for $P^{*}$ to be an optimum solution to the problem. Specifically, for $M=3$ rounds and the Lagrangian $\mathrm{L}\left(P_{1}^{*}, P_{2}^{*}, P_{3}^{*}, \lambda^{*}\right)[19]$ :

Stationarity:

$$
\frac{\partial \mathrm{L}\left(P_{1}^{*}, P_{2}^{*}, P_{3}^{*}, \lambda^{*}\right)}{\partial P_{1}^{*}}=\frac{\partial \mathrm{L}\left(P_{1}^{*}, P_{2}^{*}, P_{3}^{*}, \lambda^{*}\right)}{\partial P_{2}^{*}}=\frac{\partial \mathrm{L}\left(P_{1}^{*}, P_{2}^{*}, P_{3}^{*}, \lambda^{*}\right)}{\partial P_{3}^{*}}=0
$$

Primal feasibility:

$$
0 \leq P_{1}^{*}, P_{2}^{*}, P_{3}^{*} \leq \bar{P}, 0 \leq P_{r}\left(C_{3} \leq C_{\text {out }}\right) \leq \varepsilon_{\text {target }}
$$

Complementary slackness:

$$
\lambda^{*} \cdot\left(P_{r}\left(C_{3} \leq C_{\text {out }}\right)-\varepsilon_{\text {target }}\right)=0
$$

Dual feasibility:

$$
\lambda^{*} \geq 0
$$

where $\varepsilon_{\text {target }}$ is the target outage probability from (7).

Besides the brute-force or exhaustive search, the standard methods for solving constrained non-linear optimization problems include interior-point (IP) methods, sequential quadratic programming (SQP) or even projected gradient descent (PGD) [18]. IP and SQP require the objective and constraint functions to be twice differentiable and exhibit polynomial time complexity for linear and non-linear problems, but the strong advantage of SQP lies in its property that the initial guess and the iteration steps do not need to be feasible points [18]. SQP is an active-set method that works in two stages: Firstly, the objective function is neglected, and a feasible point is obtained that satisfies the constraints. Secondly, the objective function is optimized while keeping the feasibility. In contrast, the IP iterations must stay inside the feasible region and avoid the infeasible region, but it yields better approximations, and it is scalable [18]. 


\section{Numerical Results and Discussion}

In this section, the optimal power allocation problem for an optical LEO-to-ground downlink with HARQ schemes is solved, and a variety of simulations are carried out from which conclusive results are drawn. In our work, the MATLAB software is employed to numerically obtain a very good approximation of the global minimum of the constrained non-linear optimization problems. Specifically, the IP and SQP methods are employed, and the outcomes are validated through a brute-force search. Moreover, the average power is minimized for Type I, CC, IR HARQ schemes, and the sensitivity to the scintillation index, average path loss and target outage probability is examined. It must be clarified that we are more focused on the proposed allocation methodologies and less on the transmission characteristics.

A $M=3$ rounds HARQ protocol is considered, and an optical LEO-to-ground link is assumed under atmospheric path loss and weak scintillation conditions. The choice of $M=3$ is supported by the fact that LEO satellites have a short contact time ( $\sim 5 \mathrm{~min})$; therefore it is realistic to assume a few HARQ rounds. The wavelength is set to $\lambda=1550 \mathrm{~nm}$, the slew rate $w_{s}=0.001$, the altitudes $H_{\mathrm{OGS}}=2000 \mathrm{~m}$ and $H_{\mathrm{LEO}}=H_{\mathrm{turb}}=20 \mathrm{~km}$ and elevation angle $e=50^{\circ}$ while the ground weather conditions, i.e., wind speed, $C_{n}^{2}(z)$, were varied to obtain the scintillation indices and the corresponding coherence times using the (1). The Bufton and Hufnagel-Valley models were employed for the wind and $C_{n}^{2}(z)$. From Table 1 it is implied that the worse the scintillation effects the less coherence time is needed because the channel fluctuations are greater and rapid. Finally, the maximum transmitted power is set to $\bar{P}=1 \mathrm{~W}$ for all simulations.

Table 1. Scintillation index versus the channel coherence time for various weather conditions.

\begin{tabular}{llc}
\hline SI & $\tau_{\mathbf{0}}$ (ms) \\
\hline 0.1 & 5.2 \\
0.2 & 1.8 \\
0.3 & 1.2 \\
0.4 & 0.9 \\
0.5 & 0.8 \\
\hline
\end{tabular}

In the first simulated scenario, the impact of SI on the average total power is evaluated for the three HARQ protocols. The target outage probability, the outage capacity-bandwidth ratio, and the optical channel statistics over the three retransmission rounds are given in Table 2, while it is assumed that $\mathrm{SI}_{1}=\mathrm{SI}_{2}=\mathrm{SI}_{3}$. The proposed methodology is simulated using the IP and SQP algorithms and validated with a brute-force search. In Figure 2 the numerical results are given.

Table 2. First simulation's input parameters for the optical channels.

\begin{tabular}{cccccccc}
\hline$\varepsilon_{\text {target }}$ & $C_{\text {out }} / \boldsymbol{W}$ & $\left\langle\boldsymbol{h}_{1}\right\rangle$ & $\left\langle\boldsymbol{h}_{2}\right\rangle$ & $\left\langle\boldsymbol{h}_{3}\right\rangle$ & $\sigma_{\boldsymbol{h}_{1}}^{2}$ & $\sigma_{\boldsymbol{h}_{2}}^{2}$ & $\sigma_{\boldsymbol{h}_{3}}^{2}$ \\
\hline 0.01 & 0.5 & 1 & 1 & 1 & $0.01-0.5$ & $0.01-0.5$ & $0.01-0.5$ \\
\hline
\end{tabular}

In Figure 2, it can be observed that the higher the SI the more power is consumed by all HARQ schemes with a rate of $12 \%$ (Type I), $6 \%$ (CC) and 5.2\% (IR). Type I is the worst and results in the largest average total power and CC is moderate, while IR is the most energy-efficient HARQ protocol. At SI $=0.3$, the average total powers of Type I and CC are $47.2 \%$ and $6.8 \%$ larger than IR, respectively.

In the second scenario of simulations, the impact of $\langle h\rangle$ on the average total power is evaluated for the three HARQ protocols. The target outage probability, the outage capacitybandwidth ratio, and the optical channel statistics over the three retransmission rounds are given in Table 3, while it is assumed that $\left\langle h_{1}\right\rangle=\left\langle h_{2}\right\rangle=\left\langle h_{3}\right\rangle$. The proposed methodology 
is simulated using the IP and SQP algorithms and validated with a brute-force search. In Figure 3 the numerical results are presented.

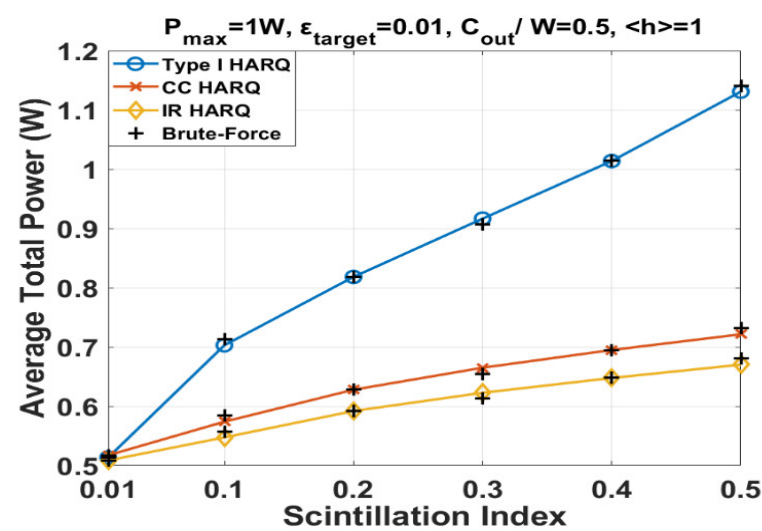

Figure 2. Optimization of average total power versus the scintillation index for Type I, CC and IR HARQ schemes.

Table 3. Second simulation's input parameters for the optical channel.

\begin{tabular}{cccccccc}
\hline$\varepsilon_{\text {target }}$ & $\boldsymbol{C}_{\text {out }} / \boldsymbol{W}$ & $\left\langle\boldsymbol{h}_{1}\right\rangle$ & $\left\langle\boldsymbol{h}_{2}\right\rangle$ & $\left\langle\boldsymbol{h}_{3}\right\rangle$ & $\sigma_{\boldsymbol{h}_{1}}^{2}$ & $\sigma_{\boldsymbol{h}_{2}}^{2}$ & $\sigma_{\boldsymbol{h}_{3}}^{2}$ \\
\hline 0.01 & 0.5 & $0.5-1$ & $0.5-1$ & $0.5-1$ & 0.1 & 0.1 & 0.1 \\
\hline
\end{tabular}

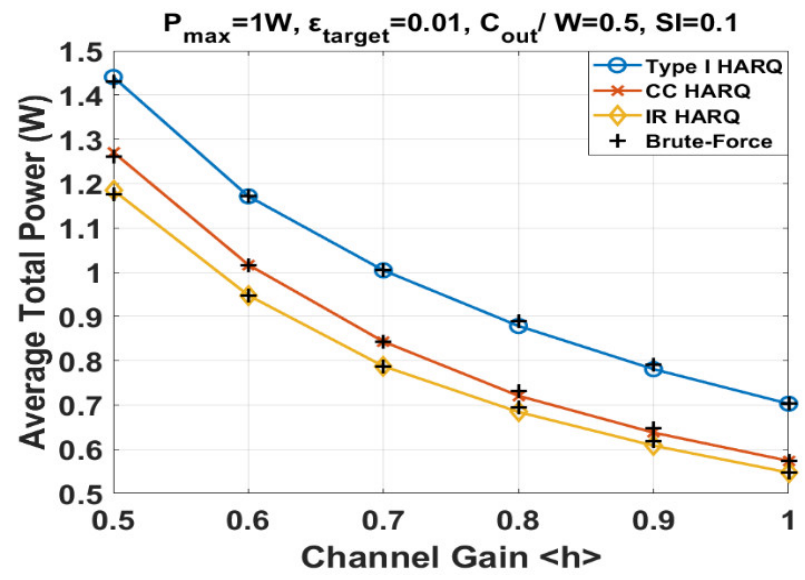

Figure 3. Optimization of average total power versus the channel gain for Type I, CC and IR HARQ schemes.

In Figure 3, it is shown that the higher the channel gain the less power is consumed by all HARQ schemes with a rate of $12.5 \%$ (Type I), $14.5 \%$ (CC) and $13.1 \%$ (IR). Type I is the worst and results in the largest average total power and CC is moderate, while IR is the most energy-efficient HARQ protocol. At $\langle h\rangle=0.8$, the average total powers of Type I and CC are $28.4 \%$ and $5.4 \%$ larger than IR, respectively.

In the third hypothetical simulation, the impact of $\varepsilon_{\text {target }}$ on the average total power is evaluated for the three HARQ protocols. The outage-capacity-to-bandwidth ratio, and the optical channel statistics over the three retransmission rounds, are given in Table 4 . The proposed methodology is simulated using the IP and SQP algorithms and validated with a brute-force search. In Figure 4 the numerical results are given. 
Table 4. Third simulation's input parameters for the optical channel.

\begin{tabular}{cccccccc}
\hline$\varepsilon_{\text {target }}$ & $\boldsymbol{C}_{\text {out }} / \boldsymbol{W}$ & $\left\langle\boldsymbol{h}_{1}\right\rangle$ & $\left\langle\boldsymbol{h}_{2}\right\rangle$ & $\left\langle\boldsymbol{h}_{3}\right\rangle$ & $\sigma_{\boldsymbol{h}_{1}}^{2}$ & $\sigma_{\boldsymbol{h}_{2}}^{2}$ & $\sigma_{\boldsymbol{h}_{3}}^{2}$ \\
\hline $10^{-7}-10^{-1}$ & 0.5 & 1 & 1 & 1 & 0.2 & 0.2 & 0.2 \\
\hline
\end{tabular}

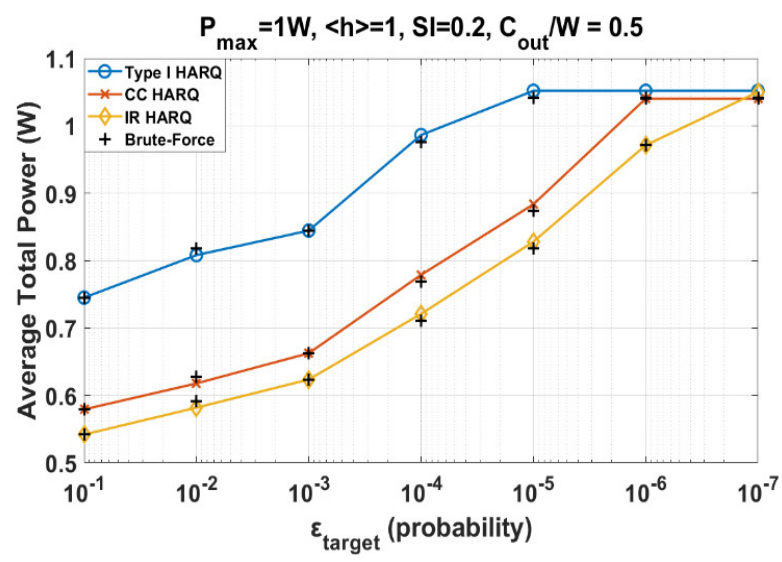

Figure 4. Optimization of average total power versus the target outage probability for Type I, CC and IR HARQ schemes.

In Figure 4, it is observed that the lesser the $\varepsilon_{\text {target}}$, the more power is consumed by all HARQ schemes with a rate of $6.7 \%$ (Type I), $13.4 \%$ (CC) and $14.8 \%$ (IR). Type I is the worst and cannot achieve an $\varepsilon_{\text {target }}<1 \times 10^{-5}$ and CC is moderate and cannot achieve an $\varepsilon_{\text {target }}<1 \times 10^{-6}$, while IR is the most energy-efficient HARQ protocol, reaching a threshold of $\varepsilon_{\text {target }}=1 \times 10^{-7}$. At $\varepsilon_{\text {target }}=1 \times 10^{-5}$, the average total powers of Type I and CC are $27 \%$ and $6.7 \%$ larger than IR, respectively.

In the fourth simulation, the channel power distribution is evaluated among the three retransmission rounds. The outage-capacity-to-bandwidth ratio, and the optical channel statistics over the three HARQ rounds, are given in Table 5. The proposed methodology is simulated using the IP and SQP algorithms and validated with a brute-force search. In Figure $5 \mathrm{a}$ the numerical results are given only for the IR HARQ protocol, which shows the best performance. In Figure $5 b$ the proposed methodologies are simulated for arbitrary input parameters given in Table 5. The average and allocated power are exhibited.

Table 5. Fourth simulation's input parameters for the optical channel.

\begin{tabular}{ccccccccc}
\hline Sim & $\varepsilon_{\text {target }}$ & $\boldsymbol{C}_{\text {out }} / \boldsymbol{W}$ & $\left\langle\boldsymbol{h}_{1}\right\rangle$ & $\left\langle\boldsymbol{h}_{2}\right\rangle$ & $\left\langle\boldsymbol{h}_{3}\right\rangle$ & $\sigma_{\boldsymbol{h}_{1}}^{2}$ & $\sigma_{\boldsymbol{h}_{2}}^{2}$ & $\sigma_{\boldsymbol{h}_{3}}^{2}$ \\
\hline (a) & $10^{-7}-10^{-1}$ & 0.5 & 1 & 1 & 1 & 0.2 & 0.2 & 0.2 \\
\hline$(\mathbf{b})$ & $10^{-4}$ & 0.44 & 0.92 & 0.97 & 0.88 & 0.24 & 0.30 & 0.22 \\
\hline
\end{tabular}

In Figure $5 \mathrm{a}$, as the $\varepsilon_{\text {target }}$ gets smaller, it can be observed that the average total power is minimized by allocating the most power on the third round and the least power during the first round until $1 \times 10^{-7}$ where maximum power is allocated over all three rounds. This is reasonable because from (23) the $P_{3} \cdot P_{r}\left(C_{2} \leq C_{\text {out }}\right)$ yields a much smaller term than $P_{2} \cdot P_{r}\left(C_{1} \leq C_{o u t}\right)$ or $P_{1}$; therefore, $P_{3}$ is allocated with maximum power first, followed by less power in $P_{2}$ and finally by the least power in $P_{1}$. In Figure $5 \mathrm{~b}$ it can be observed that CC and IR perform much more efficiently than Type I, and in a similar way, by allocating more power on the first and third rounds. It must be mentioned that not all $M$ re-transmissions are required; the proposed methodologies simply indicate the gradual increment of allocated power till the successful decoding or depletion of HARQ rounds [15]. 


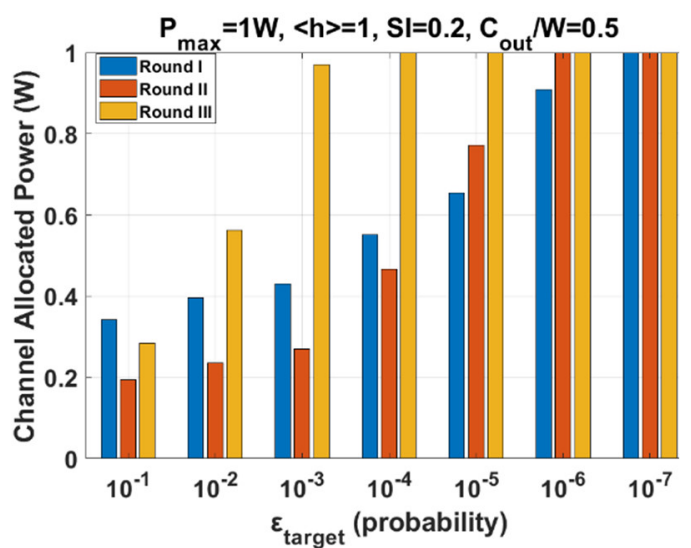

(a)

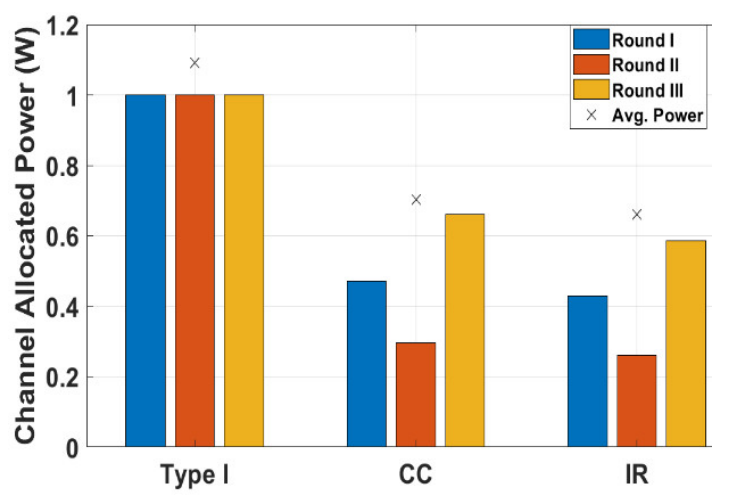

(b)

Figure 5. (a) Channel-allocated power versus the target outage probability for the IR HARQ protocol; (b) channel-allocated power and average power of the three HARQ schemes for arbitrary input channel parameters.

Lastly, a comparison between IP and SQP algorithms has been implemented and shown for the previous case of IR HARQ. The stopping criteria, the termination tolerances of the constraints, the step sizes and the initial power vector $P^{(0)}$ are given in Table 6 . In Tables 7 and 8 the algorithms' performance parameters are reported.

Table 6. Optimization parameters of IP and SQP algorithms.

\begin{tabular}{ccccc}
\hline & Stopping Criterion & Termination Tolerance & Step Size & Initial Vector \\
\hline IP & 1000 iterations & $1 \times 10^{-6}$ & $1 \times 10^{-10}$ & $P^{(0)}=\left[\begin{array}{lll}0.5 & 0.5 & 0.5\end{array}\right]^{T} \mathrm{~W}$ \\
$\mathrm{SQP}$ & 400 iterations & $1 \times 10^{-6}$ & $1 \times 10^{-6}$ & $P^{(0)}=\left[\begin{array}{lll}0.5 & 0.5 & 0.5\end{array}\right]^{T} \mathrm{~W}$ \\
\hline
\end{tabular}

Table 7. Performance Parameters of Interior-Point Algorithm.

\begin{tabular}{ccccc}
\hline$\varepsilon_{\text {target }}$ & Iterations & $\begin{array}{c}\text { Function } \\
\text { Evaluations }\end{array}$ & $\begin{array}{c}\text { 1st Order } \\
\text { Optimality }\end{array}$ & $\begin{array}{c}\text { Constraint } \\
\text { Violations }\end{array}$ \\
\hline $1 \times 10^{-1}$ & 13 & 58 & $3.8 \times 10^{-8}$ & 0 \\
$1 \times 10^{-2}$ & 12 & 53 & $4 \times 10^{-7}$ & 0 \\
$1 \times 10^{-3}$ & 20 & 91 & $9.3 \times 10^{-7}$ & 0 \\
$1 \times 10^{-4}$ & 22 & 101 & $1.6 \times 10^{-8}$ & 0 \\
$1 \times 10^{-5}$ & 29 & 131 & $3.2 \times 10^{-7}$ & 0 \\
$1 \times 10^{-6}$ & 29 & 133 & $8 \times 10^{-8}$ & 0 \\
$1 \times 10^{-7}$ & 30 & 139 & $5.7 \times 10^{-7}$ & $5.7 \times 10^{-7}$ \\
\hline
\end{tabular}

Table 8. Performance Parameters of Sequential Quadratic Programming Algorithm.

\begin{tabular}{ccccc}
\hline$\varepsilon_{\text {target }}$ & Iterations & $\begin{array}{c}\text { Function } \\
\text { Evaluations }\end{array}$ & $\begin{array}{c}\text { 1st Order } \\
\text { Optimality }\end{array}$ & $\begin{array}{c}\text { Constraint } \\
\text { Violations }\end{array}$ \\
\hline $1 \times 10^{-1}$ & 10 & 45 & $3.5 \times 10^{-7}$ & $2.7 \times 10^{-17}$ \\
$1 \times 10^{-2}$ & 9 & 43 & $6.5 \times 10^{-7}$ & $3.1 \times 10^{-11}$ \\
$1 \times 10^{-3}$ & 8 & 37 & $7.3 \times 10^{-7}$ & $2.4 \times 10^{-11}$ \\
$1 \times 10^{-4}$ & 8 & 36 & $1.9 \times 10^{-8}$ & $8.2 \times 10^{-15}$ \\
$1 \times 10^{-5}$ & 10 & 44 & $1.6 \times 10^{-7}$ & $1.4 \times 10^{-17}$ \\
$1 \times 10^{-6}$ & 12 & 52 & $6.7 \times 10^{-7}$ & $3 \times 10^{-15}$ \\
$1 \times 10^{-7}$ & 9 & 68 & $8.6 \times 10^{-7}$ & $8.6 \times 10^{-7}$ \\
\hline
\end{tabular}

Although the two algorithms (IP and SQP) yield the same numerical results in all the examined cases, as exhibited previously in Figures 1-4, they require a different number 
of iterations and function evaluations. Additionally, they converge to a local minimum with different first-order optimality and constraint violations. The first-order optimality translates to the maximum absolute value (infinity norm) of the gradient of the Lagrangian, and ideally it should be zero at the minimum. The constraint violations refer to the nonlinear target outage probability constraints and ideally, they should be zero.

From Tables 1 and 2 it is observed that IP algorithm requires approximately 3 times more iterations and function evaluations than SQP, especially at $\varepsilon_{\text {target }}=1 \times 10^{-7}$, which means that IP is slower. This outcome is reasonable since IP by definition is required to stay inside the feasible region and bounds at all iterations, while SQP allows some constraint and bound violations. On the other hand, IP achieves better first-order optimality has almost no constraint violations, which makes IP a better approximation of the local minimum than SQP.

\section{Conclusions}

In this paper, optical HARQ-based LEO-to-ground downlinks under weak turbulence conditions are studied, and a power allocation methodology is proposed to ensure reliable and energy-efficient transmission. Specifically, to optimize the system's energy efficiency and reliability, the average power consumption is minimized given a maximum transmitted power constraint and a target outage probability constraint. Assuming a finite number of rounds with temporal spacing between retransmissions of at least a channel's coherence time, the optimization problem is constructed as a constrained nonlinear programming problem for the cases of Type I, CC and IR HARQ schemes. The optical channel's statistics are required to obtain the optimal power allocation rather than the instantaneous channel gains. The solutions are derived numerically via iterative algorithms, namely IP and SQP, and validated through a brute-force search, i.e., a search of all points in the feasible region. Simulations are then executed to evaluate the proposed methodology and provide performance parameters of IP and SQP while also exhibiting the impact of SI, average path loss and target outage probability. The numerical results show that Type I HARQ yields the highest average total power, CC is moderate and IR yields the lowest average total power. The IP algorithm was found to be more accurate in finding the global solution because of its nearly zero first-order optimality and constraint violations, but SQP is faster due to the smaller number of iterations and function evaluations. For future work, the impact of correlation between the channels of the retransmission rounds can be investigated. The case of strong turbulence (SI $>1$ ) for smaller elevation angles may also be studied. Finally, real experimental data from optical LEO-to-ground can be employed to evaluate the feasibility of HARQ schemes.

Author Contributions: Conceptualization, T.T.K. and A.D.P.; methodology, T.T.K. and A.D.P.; software, T.T.K.; validation, T.T.K. and A.D.P.; formal analysis, T.T.K. and A.D.P.; investigation, T.T.K. and A.D.P.; resources, T.T.K. and A.D.P.; writing—original draft preparation, T.T.K.; writing-review and editing, T.T.K. and A.D.P.; visualization, T.T.K.; supervision, A.D.P.; funding acquisition, T.T.K. and A.D.P. All authors have read and agreed to the published version of the manuscript.

Funding: This research received no external funding.

Data Availability Statement: Not applicable.

Conflicts of Interest: The authors declare no conflict of interest.

\section{References}

1. Chaudhry, A.U.; Yanikomeroglu, H. Free Space Optics for Next-Generation Satellite Networks. IEEE Consum. Electron. Mag. 2021, 10, 21-31. [CrossRef]

2. Uysal, M.; Capsoni, C.; Ghassemlooy, Z.; Boucouvalas, A.; Udvary, E. Signals and Communication Technology Optical Wireless Communications-An Emerging Technology; Springer International Publishing: Cham, Switzerland, 2016; pp. 1-639. [CrossRef]

3. Kaushal, H.; Kaddoum, G. Free Space Optical Communication: Challenges and Mitigation Techniques. IEEE Commun. Surv. Tutor 2016, 19, 57-96. [CrossRef]

4. Hemmati, H. Near-Earth Laser Communications Edited By; CRC Press: Boca Raton, FL, USA, 2009. 
5. Dosti, E.; Shehab, M.; Alves, H.; Latva-Aho, M. Ultra Reliable Communication via Optimum Power Allocation for HARQ Retransmission Schemes. IEEE Access 2020, 8, 89768-89781. [CrossRef]

6. Hosseini, S.S.; Abouei, J.; Champagne, B.; Chang, X.W. A Novel Cooperative HARQ Protocol for Free-Space Optical Broadcasting Systems. J. Lightwave Technol. 2020, 38, 1789-1799. [CrossRef]

7. Pan, G.; Ye, J.; Tian, Y.; Alouini, M.S. On Harq Schemes in Satellite-Terrestrial Transmissions. IEEE Trans. Wirel. Commun. 2020, 19, 7998-8010. [CrossRef]

8. Kiasaleh, K. Hybrid ARQ for FSO Communications through Turbulent Atmosphere. IEEE Commun. Lett. 2010, 14, 866-868. [CrossRef]

9. Kolev, D.R.; Toyoshima, M. Satellite-to-Ground Optical Communications Using Small Optical Transponder (SOTA)—ReceivedPower Fluctuations. Opt. Express 2017, 25, 28319-28329. [CrossRef]

10. Tokovinin, A.; Kellerer, A.; du Foresto, V.C. FADE, an Instrument to Measure the Atmospheric Coherence Time. Astron. Astrophys. 2008, 477, 671-680. [CrossRef]

11. Zedini, E.; Chelli, A.; Alouini, M.S. Unified Performance Analysis of Hybrid-ARQ with Incremental Redundancy over Free-Space Optical Channels. In Proceedings of the IEEE 25th Annual International Symposium on Personal, Indoor, and Mobile Radio Communication (PIMRC), Washington, DC, USA, 1-2 September 2014; pp. 774-778. [CrossRef]

12. Zedini, E.; Chelli, A.; Alouini, M.S. On the Performance Analysis of Hybrid ARQ With Incremental Redundancy and With Code Combining Over Free-Space Optical Channels With Pointing Errors. IEEE Photonics J. 2014, 6, 1-18. [CrossRef]

13. Chaitanya, T.V.K.; Larsson, E.G. Outage-Optimal Power Allocation for Hybrid ARQ with Incremental Redundancy. IEEE Trans. Wirel. Commun. 2011, 10, 2069-2074. [CrossRef]

14. Su, W.; Lee, S.; Pados, D.A. The Optimal Transmission Power Per Round for Hybrid-ARQ Rayleigh Fading Links. In Proceedings of the IEEE International Conference on Communications, Cape Town, South Africa, 23-27 May 2010; pp. 1-5. [CrossRef]

15. Dosti, E.; Charalambous, T.; Wichman, R. Power Allocation for ARQ Two-Hop Cooperative Networks for Ultra-Reliable Communication. In Proceedings of the IEEE International Conference on Communications (ICC), Dublin, Ireland, 7-14 June 2020; pp. 1-7. [CrossRef]

16. Chen, Y.-A.; Zhang, Q.; Chen, T.-Y.; Cai, W.-Q.; Liao, S.-K.; Zhang, J.; Chen, K.; Yin, J.; Ren, J.-G.; Chen, Z.; et al. An integrated space-to-ground quantum communication network over 4600 kilometres. Nat. Cell Biol. 2021, 589, 214-219.

17. Ntanos, A.; Lyras, N.K.; Zavitsanos, D.; Giannoulis, G.; Panagopoulos, A.D.; Avramopoulos, H. LEO Satellites Constellation-toGround QKD Links: Greek Quantum Communication Infrastructure Paradigm. Photonics 2021, 8, 544. [CrossRef]

18. Nocedal, J.; Wright, S.J. Numerical Optimization Second Edition; Springer Science \& Business Media LLC: New York, NY, USA, 2006.

19. Boyd, S.P. Vandenberghe, Lieven. In Convex Optimization; Cambridge University Press: Cambridge, UK, 2004.

20. Kolev, D.R.; Toyoshima, M. Received-Power Fluctuation Analysis for LEO Satellite-to-Ground Laser Links. J. Lightwave Technol. 2017, 35, 103-112. [CrossRef]

21. Kapsis, T.T.; Panagopoulos, A.D. Optimum Power Allocation Based on Channel Conditions in Optical Satellite Downlinks. Wireless Pers. Commun. 2021, 116, 2997-3013. [CrossRef] 